\title{
Oxypaeoniflorin improves myocardial ischemia/reperfusion injury by activating the Sirt1/Foxo1 signaling pathway
}

\author{
Kai Wang ${ }^{1}$ and Wei $\mathrm{Hu}^{2} \bowtie$ \\ 1Department of TCM Pharmacy, Ningbo Medical Center Li Huili Hospital; ²Department of TCM Pharmacy, Huamei Hospital, Haishu District, \\ Ningbo, Zhejiang Province, China Academy of Sciences
}

\begin{abstract}
Myocardial ischemia/reperfusion (MI/R) injury is a leading cause of damage to cardiac tissues and is associated with high mortality and disability rates worldwide. Oxypaeoniflorin (OPA) has been found to be the main constituent of Paeonia veitchii Lynch. This study was conducted to explore the effect of OPA on MI/R injury and its potential mechanism. An in vivo $M I / R$ injury model was established by transient coronary ligation in $B A L B / C$ mice, and an in vitro hypoxia/reoxygenation (H/R) injury model was established with rat cardiomyocyte $\mathrm{H} 9 \mathrm{c2}$ cells. Echocardiographic assessments demonstrated that OPA significantly reduced disruption of cardiac function and improved the indicators of ejection fraction (EF) and fractional shortening (FS). The enzyme-linked immunosorbent assay (ELISA) results suggested that OPA significantly reduced the release of myocardial infarction-related factors, such as the creatine kinase (CK-MB), cardiac troponin I (cTnl) and cardiac troponin T (cTnT). Additionally, hematoxylin-eosin (H\&E) staining demonstrated that OPA markedly inhibited the myocardial apoptosis and necrosis caused by MI/R. Consistently, the results obtained from the cell counting kit-8 (CCK-8) and flow cytometry assays revealed that OPA obviously reversed the $H / R$-induced decrease in cell activity and increase in apoptosis of $\mathrm{H} 9 \mathrm{c} 2$ cells. Furthermore, western blot assays indicated that OPA inhibited apoptosis by activating the Sirt 1 (silent information regulator factor 2 related enzyme 1)/Foxo1(forkhead transcription factor FKHR) signaling pathway in myocardial tissues and $\mathrm{H} 9 \mathrm{c} 2$ cells. Collectively, these novel findings are the first to provide strong evidence that OPA attenuates MI/R injury by activating the Sirt1 (silent information regulator factor 2 related enzyme 1)/Foxo1(forkhead transcription factor FKHR) signaling-mediated anti-apoptotic pathway.
\end{abstract}

Key words: Oxypaeoniflorin, myocardial ischemia/reperfusion, apoptosis, Sirt1/Foxo1

Received: 18 March, 2020; revised: 26 April, 2020; accepted: 20 May, 2020; available on-line: 18 June, 2020

$\square$ e-mail: qiuyuyan222@163.com

Abbreviations: CCK-8, cell counting kit-8; CK-MB, creatine kinase; cTnl, cardiac troponin I; cTnT, cardiac troponin T; EF, ejection frac tion; ELISA, immunosorbent assay; H/R, hypoxia/reoxygenation; FS, fractional shortening; MI/R, Myocardial ischemia/reperfusion

\section{INTRODUCTION}

Ischemic heart disease, also known as coronary atherosclerotic heart disease (referred to as coronary heart disease), has become the leading cause of death worldwide (Finegold et al., 2013). According to the World Health Organization, coronary heart disease caused a total of 7.4 million deaths worldwide in 2012, and its incidence has increased annually in China (Zhu et al., 2016). Mounting evidence has demonstrated that the occlusion of blood vessels by coronary arteriosclerotic plaques due to myocardial ischemia can lead to myocardial tissue damage; after the vascular occlusion is removed, the process of regaining blood supply to the myocardial tissue causes further myocardial tissue damage, which is widely known as myocardial ischemia/reperfusion (MI/R) injury (Buja, 2013). MI/R injury can lead to many adverse complications, including cardiomyocyte necrosis and apoptosis, ultimately destroying the cardiac contractile function (Salas et al., 2010; Wang et al., 2019). Naturally, protecting cardiomyocytes from apoptosis and necrosis could be a reasonable method of ameliorating the MI/R injury (Wang et al., 2015; Zhu et al., 2015; Xu et al., 2019; Wang et al., 2016). Therefore, for the prevention and treatment of ischemic heart disease, it is of great significance to explore the mechanisms that regulate $\mathrm{MI} / \mathrm{R}$ injury and those that protect against $\mathrm{MI} / \mathrm{R}$ injury.

Paeonia lactiflora Pall. is a well-known Chinese herbal medicine of the Ranunculaceae family that is used to relieve abdominal cramps, reduce pain and improve blood circulation (Chen et al., 2013). The main active chemical components of Paeonia lactiflora include paeoniflorin and oxypaeoniflorin. Among these components, paeoniflorin has been reported to protect against the $\mathrm{MI} / \mathrm{R}$ injury by inhibiting inflammation and apoptosis (Nizamutdinova et al., 2008; Qian et al., 2015; Chen et al., 2015; Li et al., 2012; Han et al., 2016b). Oxypaeoniflorin (OPA), also known as [1a-(hexopyranosyloxy)-5-hydroxy-2-methyltetrahydro-1H-2,5-methano-3,4-dioxacyclobuta[cd]pentalen$5 \mathrm{~b}(3 \mathrm{aH})-y \mathrm{l}]$ methyl 4-hydroxybenzoate, has a molecular formula of $\mathrm{C}_{23} \mathrm{H}_{28} \mathrm{O}_{12}$ and a molecular weight of 496.46 (Feng et al., 2010). OPA is a monoterpene glycoside compound very similar to paeoniflorin and has been reported to alleviate advanced glycation end product-induced oxidative damage and inflammation in mesangial cells (Zhang et al., 2013). In addition, a recent study revealed that OPA attenuated inflammatory effects via regulation of the toll-like receptor (TLR), extracellular signal-related kinase (ERK) and p38 mitogen-activated protein (MAP) kinases signaling pathways in LPS-stimulated RAW264.7 cells (Yoo et al., 2018b). However, the effect of OPA on MI/R injury remains unclear and needs to be clarified and studied. Based on the data described above, we hypothesized that OPA could alleviate the MI/R injury. Therefore, $\mathrm{MI} / \mathrm{R}$ injury models were designed to investigate the effects of OPA on the apoptosis of cardiomyocytes both, in vivo and in vitro, and studies of 


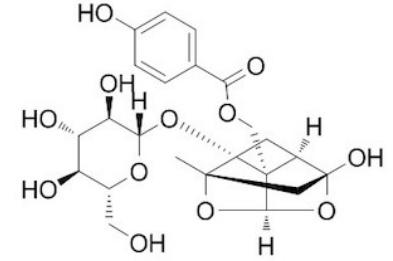

Figure 1. Chemical structure of oxypaeoniflorin (OPA).

the molecular mechanism focused on the Sirt1/Foxo1 signaling.

\section{MATERIALS AND METHODS}

Reagents. Oxypaeoniflorin (purity $>98 \%$ ) was purchased from Shanghai Hengfei Biological Technology Co., Ltd., and the molecular structure is shown in Fig. 1. The antibodies against Sirt1 (\#ab12193), Bcl-2 (\#ab59348), Bax (\#ab32503), caspase-3 (\#ab13847), cleaved caspase-3 (\#ab49822), and GAPDH (\#ab49822) and the HRP-conjugated goat antirabbit IgG (\#ab6721) were all purchased from Abcam (Cambridge, UK). The antibodies against acetylated Foxo1 (Ac-Foxo1, \#sc-49437) were obtained from Santa Cruz Biotechnology (Santa Cruz, CA, USA). Dulbecco's modified Eagle's medium, penicillin, streptomycin, and fetal bovine serum were all purchased from Sigma Aldrich (St. Louis, MO, USA). An enzyme-linked immunosorbent assay kit was purchased from Thermo Fisher Scientific (Waltham, MA, USA). A hematoxylin and eosin staining kit, CCK-8 solution, BCA detection kit and ECL reagent were purchased from Beyotime Biotechnology (Shanghai, China). The Annexin V-FITC/PI Apoptosis Detection Kit was purchased from Becton Dickinson (Rutherford, NJ, USA). The reagents used to make the ischemic buffer (137 mM NaCl, $12 \mathrm{mM} \mathrm{KCl,} 0.49 \mathrm{mM} \mathrm{MgCl}, 0.9 \mathrm{mM}$ $\mathrm{CaCl}_{2}, 4.0 \mathrm{mM}$ HEPES, $10.0 \mathrm{mM}$ deoxyglucose, 0.75 $\mathrm{mM}$ sodium dithionate, and $20.0 \mathrm{mM}$ lactate) were all of analytical or reagent grade.

Animals. A total of 30 C57BL/ 6 male mice, 6-8 weeks of age (body weight 20-25 g), were obtained from the Shanghai Slack Laboratory Animal Co., Ltd. (production license no. CXK(HU)2017-0015), raised in the animal experimental research center of ZheJiang Chinese Medical University, and used in this study. All the procedures were performed in accordance with the Guidelines for the Care and Use of Laboratory Animals published by the U.S. National Institutes of Health $(\mathrm{NIH}$ publication NO. 85-23, revised in 1996). The protocol was reviewed and approved by the Ethical Committee of ZheJiang Chinese Medical University before the animal study (No. ZSLL-2017-059). All the mice were maintained in diurnal lighting conditions $(12 \mathrm{~h} / 12 \mathrm{~h})$ at a constant temperature of $25 \pm 2{ }^{\circ} \mathrm{C}$ for 7 days before the experiment.

In vivo $\mathrm{MI} / \mathrm{R}$ model and treatment. The $\mathrm{MI} / \mathrm{R}$ injury model was established as previously described (Yu et al., 2014; Guan et al., 2016). In brief, a mouse model of $\mathrm{MI} / \mathrm{R}$ injury was established by ligation of the left anterior descending coronary artery for 0.5 hours, followed by reperfusion for 2 hours. The mice in the sham operation group were not ligated. The mice were randomly assigned to the following groups (6 mice for each group): (1) Sham group; (2) MI/R group; (3) $\mathrm{MI} / \mathrm{R}+\mathrm{OPA}(10 \mathrm{mg} / \mathrm{kg}$ ) group; (4) MI/R + OPA (20 mg/kg) group, and (5) MI/R + OPA (40 $\mathrm{mg} / \mathrm{kg}$ ) group. OPA was administered intragastrically at the concentrations described above (diluted in sterile saline containing less than $1 \%$ dimethyl sulfoxide (DMSO)) every day for 30 days before the surgery. Finally, blood samples from the sacrificed mice were collected for biochemical analysis, and the hearts of the sacrificed rats were collected for pathological and biochemical examination.

In vitro $H / R$ cell model and treatment. $H 9 c 2$ cells were purchased from American Type Culture Collection (ATCC, Rockville, MD) and cultured in Dulbecco's modified Eagle's medium (Sigma-Aldrich, USA) with 1\% penicillin/streptomycin and 10\% fetal bovine serum (Sigma-Aldrich, USA). To establish the $\mathrm{H} / \mathrm{R}$ injury model, the procedure was performed as previously described $(\mathrm{Li}$ et al., 2017). Briefly, H9c2 cells were cultured in an ischemic buffer. The $\mathrm{pH}$ of the buffer was 6.5 , and the cells were incubated for 2 hours in a humidified cell incubator containing $21 \%$ oxygen and $5 \% \mathrm{CO}_{2}$ at $37^{\circ} \mathrm{C}$. Reperfusion was initiated by culturing the cells in normal medium for 4 hours under the above incubation conditions. Then, the cells were treated with OPA at different concentrations, and the experimental groups were as follows: (1) Control group; (2) H/R group; (3) H/R group $+0.1 \mu \mathrm{M}$ OPA; (4) H/R group $+1 \mu \mathrm{M}$ OPA; and (5) $\mathrm{H} / \mathrm{R}$ group $+10 \mu \mathrm{M}$ OPA. The cells were cultured for 8 hours in serum-free medium under the conditions described above before establishing the ischemia-reperfusion injury model.

Echocardiography. Two-dimensional echocardiography was performed using the VisualSonics Vevo 770 machine (VisualSonics, Toronto, ON, Canada) as previously described (Zhang et al., 2018). In brief, the mice were anesthetized by inhalation of $1.5 \%$ isoflurane 72 hours after MI/R surgery, and motion (M)-mode echocardiographic images were used to determine the left ventricular end systolic diameter (LVESD) and left ventricular end diastolic diameter (LVEDD). The ejection fraction (EF) and fractional shortening (FS) were calculated with the following equations:

$\mathrm{EF}(\%)=\left[\left(\mathrm{LVEDD}^{3}-\mathrm{LVESD}^{3}\right) / \mathrm{LVEDD}^{3}\right] \times 100$

FS $(\%)=[($ LVEDD - LVESD $) /$ LVEDD $] \times 100$

Detection of the myocardial enzyme levels in the serum. The mice were sacrificed after the peripheral blood was collected, and serum was separated to evaluate indicators of the heart muscle damage, including creatine kinase (CK-MB), cardiac troponin I (cTnI) and cardiac troponin $\mathrm{T}$ (cTnT), using an enzyme-linked immunosorbent assay kit (Thermo Fisher Scientific, USA).

Hematoxylin-eosin (H\&E) staining. Two hours after MI/R surgery, the chests of mice were opened again. The hearts were separated from the aortic roots and washed with prechilled phosphate-buffered saline at $4^{\circ} \mathrm{C}$, followed by fixation in $4 \%$ paraformaldehyde for 24 hours. Then, the hearts were embedded and cut into $4-\mu \mathrm{m}$ sections by a paraffin slicing machine (Leica Microsystems, Germany). For H\&E staining, the sections were deparaffinized in xylene, hydrated in serially diluted ethanol, and stained with a hematoxylin and eosin staining kit for $10 \mathrm{~min}$. Representative photomicrographs were captured by a light microscope (Olympus Corp. Tokyo, Japan). 
A
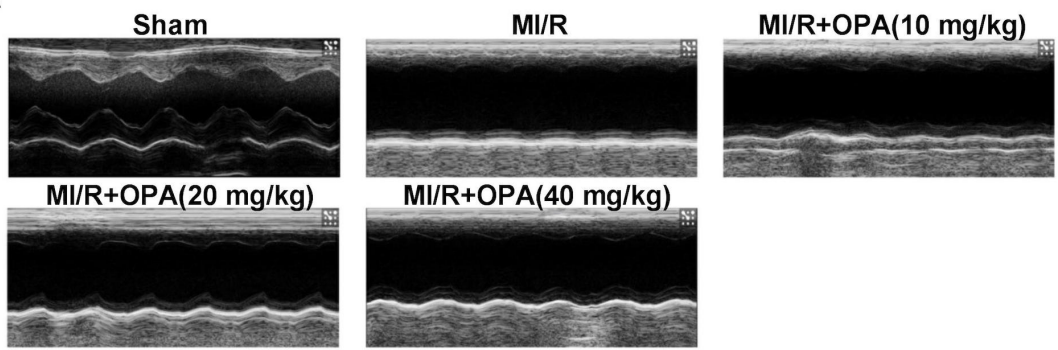

$\mathrm{Ml} / \mathrm{R}+\mathrm{OPA}(40 \mathrm{mg} / \mathrm{kg})$

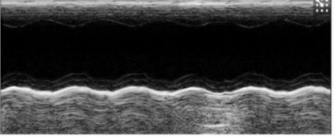

B

C
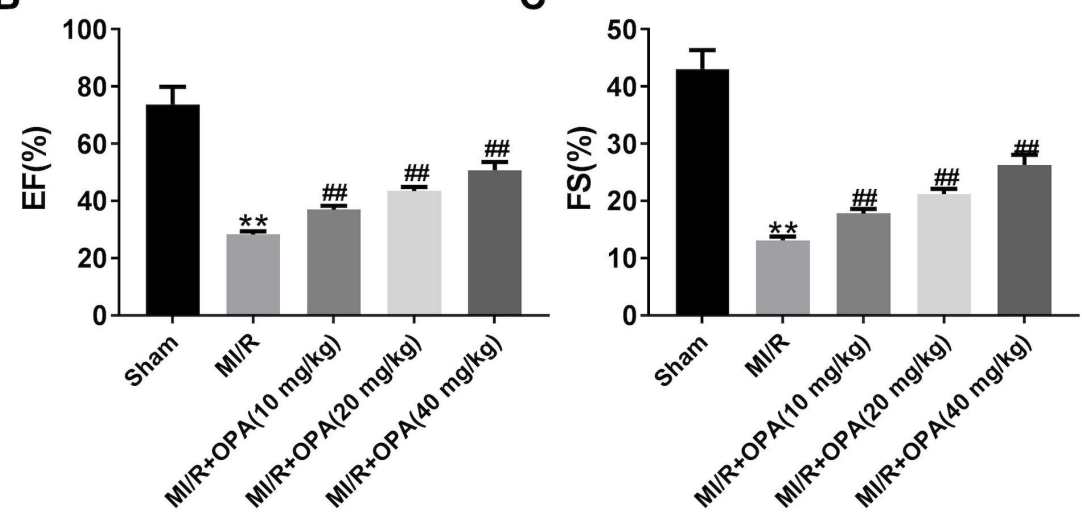

Figure 2. Effects of OPA on the cardiac functions in MI/R mice observed by echocardiographic assessment.

(A) Representative motion (M)-mode images obtained by echocardiography. (B) Ejection fraction (EF). (C) Fractional shortening (FS). ${ }^{*} P<0.05$, ${ }^{* *} P<0.01$ compared with the Sham group; ${ }^{*} P<0.05$, ${ }^{\#} P<0.01$ compared with the MI/R group.

Cell viability. Cultured H9c2 cells $\left(1 \times 10^{4}\right.$ per well $)$ were plated into 96-well plates. Ten microliters of CCK8 solution was added to each well after reperfusion, which was followed by incubation for $4 \mathrm{~h}$; then, the absorbance was measured at $450 \mathrm{~nm}$ using a microplate reader.

Flow cytometry. The Annexin V-FITC/PI Apoptosis Detection Kit was used to quantify cell apoptosis. The cells were resuspended in $200 \mu \mathrm{L}$ Annex binding buffer with $10 \mu \mathrm{L}$ Annexin V-FITC and $5 \mu \mathrm{L} \mathrm{PI}$, in the dark for $10 \mathrm{~min}$ at $25^{\circ} \mathrm{C}$. Flow cytometry (Abcam, USA) was used to analyze the double-stained cells.

Western blot. The proteins were obtained from the tissues and cells, and the concentration was determined with a BCA detection kit. A total of $30 \mu \mathrm{g}$ protein was separated by SDS-PAGE and transferred onto PVDF membranes (Millipore, USA). After blocking in 5\% skim milk for $1 \mathrm{~h}$, the membranes were incubated in primary antibodies, such as anti-Sirt1 (1:2000), anti-Ac-FoxO1 $(1: 500)$, anti-Bcl-2 (1:800), anti-Bax (1:2000), anti-caspase-3 (1:500), anti-cleaved caspase-3 (1:500), and antiGAPDH $(1: 2500)$, at $4^{\circ} \mathrm{C}$ overnight. Then, the membranes were incubated with HRP-conjugated goat antirabbit $\operatorname{IgG}(1: 2000)$ for $45 \mathrm{~min}$ at room temperature. Finally, the bands were examined with an ECL reagent. The signals were analyzed via Image Lab ${ }^{\text {TM }}$ Software (NIH Image, Bethesda, USA).

Statistical analyses. The GraphPad 7.0 software was used to analyze the data. All the experiments were repeated at least three independent times, and the data are expressed as the mean \pm standard deviation (SD). Student's $t$-test and one-way ANOVA were used to compare the significance of the differences among experi- mental groups. $(* P<0.05, * * P<0.01, * * * P<0.001)$, and $P<0.05$ were considered significant.

\section{RESULTS}

Effects of OPA on the cardiac functions in MI/R mice observed by echocardiographic assessment

To investigate the effect of OPA on the cardiac function of $\mathrm{MI} / \mathrm{R}$ mice, the left ventricular end systolic diameter (LVESD) and left ventricular end diastolic diameter (LVEDD) were measured by echocardiography to calculate the ejection fraction (EF) and fractional shortening (FS), which are indexes of LV systolic function. The results shown in Fig. 2A-C confirm that the mouse model of $\mathrm{MI} / \mathrm{R}$ was successfully established, as shown by the following evidence. Compared to the mice in the Sham group, the mice in the MI/R group showed a decreased cardiac function, and the EF and FS of the MI/R group were decreased by approximately $40.48 \%$ and $29.98 \%$, respectively (Fig. 2A-C). In addition, OPA $(10,20$ and $40 \mathrm{mg} / \mathrm{kg}$ ) observably reversed the abnormal EF and FS values in the mice subjected to $\mathrm{MI} / \mathrm{R}$ injury in a dose-dependent manner (Fig. 2A-C). Specifically, compared with those in the MI/R group, the EF and FS in the MI/R+OPA $(10 \mathrm{mg} / \mathrm{kg})$ group increased by approximately $8.72 \%$ and $4.72 \%$, the EF and $\mathrm{FS}$ in the MI/R+OPA $(20 \mathrm{mg} / \mathrm{kg})$ group increased by approximately $15.16 \%$ and $8.10 \%$, and the EF and FS in the $\mathrm{MI} / \mathrm{R}+\mathrm{OPA}$ (40 $\mathrm{mg} / \mathrm{kg}$ ) group increased by approximately $22.55 \%$ and $13.21 \%$, respectively. These data demonstrated that the MI/R injury model was suc- 

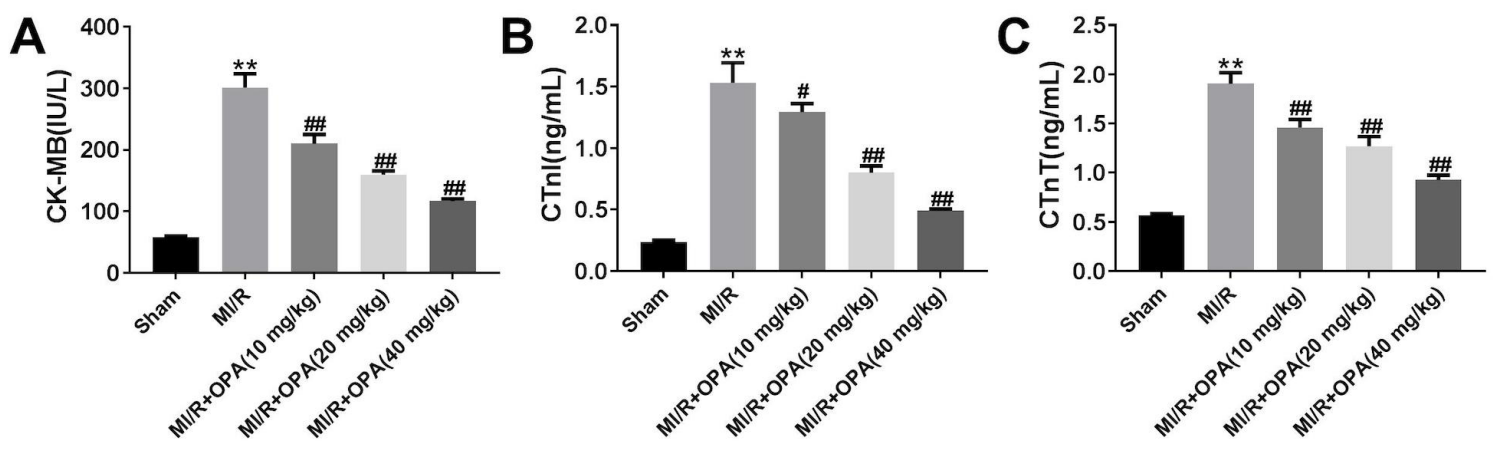

Figure 3. Effects of OPA on the myocardial enzyme markers in MI/R mice.

(A) Serum creatine kinase-MB (CK-MB) levels. (B) Serum cardiac troponin I (cTnl) levels. (C) Serum cardiac troponin T (cTnT) levels. ${ }^{*} P<0.05$, ${ }^{* *} P<0.01$ compared with the Sham group; ${ }^{\# P}<0.05$, ${ }^{*} P<0.01$ compared with the MI/R group.

cessfully established and that OPA could significantly improve cardiac function in the $\mathrm{MI} / \mathrm{R}$ mice.

\section{Effects of OPA on the myocardial enzyme markers in MI/R mice}

To further explore the protective effects of OPA against $\mathrm{MI} / \mathrm{R}$ injury, the levels of the myocardial enzyme CK-MB and the structural proteins cTnI and c'TnT were evaluated in the serum of mice in different groups (Fig. 3A-C). Significantly increased levels of CK-MB, cTnI and cTnT were observed in the $\mathrm{MI} / \mathrm{R}$ group compared to those in the Sham group. Compared with those in the Sham group, the levels of $\mathrm{CK}-\mathrm{MB}$, cTnI and c'TnT in the MI/R group were significantly increased by $243.48 \mathrm{IU} / \mathrm{L}, 1.29 \mathrm{ng} / \mathrm{mL}$ and $1.34 \mathrm{ng} / \mathrm{mL}$, respectively. OPA treatment dramatically reduced the $\mathrm{MI} / \mathrm{R}$-induced increase in the levels of $\mathrm{CK}-\mathrm{MB}$, c'TnI and c'TnT in a dose-dependent manner. Specifically, compared with those in the MI/R group, the levels of CK-MB, c'TnI and c'TnT in the $\mathrm{MI} / \mathrm{R}+\mathrm{OPA}(10 \mathrm{mg} / \mathrm{kg})$ group decreased by approximately $91.25 \mathrm{IU} / \mathrm{L}, 0.24 \mathrm{ng} / \mathrm{mL}$ and $0.44 \mathrm{ng} / \mathrm{mL}$, the levels of CK-MB, cTnI and cTnT in the MI/R+OPA $(20 \mathrm{mg} / \mathrm{kg})$ group decreased by approximately 141.66 $\mathrm{IU} / \mathrm{L}, 0.73 \mathrm{ng} / \mathrm{mL}$ and $0.63 \mathrm{ng} / \mathrm{mL}$, and the levels of $\mathrm{CK}-\mathrm{MB}$, cTnI and cTnT in the MI/R+OPA (20 mg/ $\mathrm{kg}$ ) group decreased by approximately $191.47 \mathrm{IU} / \mathrm{L}$,

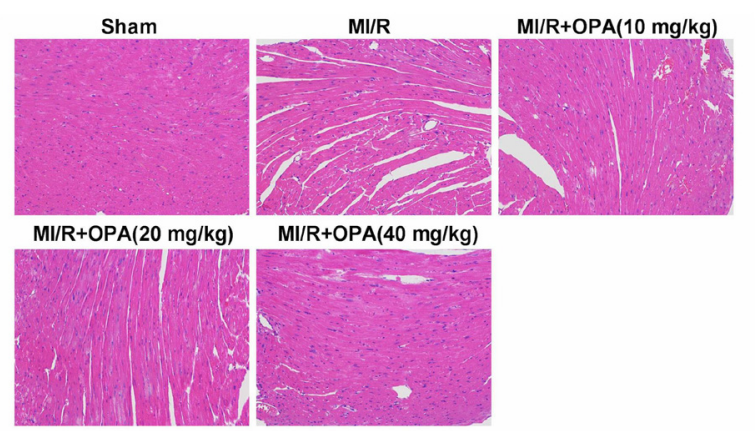

Figure 4. Effects of OPA on the histochemical alterations in MI/R mice.

Histopathological changes in mouse cardiac tissues observed by H\&E staining (200x, bar $=100 \mu \mathrm{m})$.

$1.04 \mathrm{ng} / \mathrm{mL}$ and $0.98 \mathrm{ng} / \mathrm{mL}$, respectively. These results suggested that OPA could ameliorate cardiac damage in the $\mathrm{MI} / \mathrm{R}$ mice.

\section{Effects of OPA on the histochemical alterations in MI/R} mice

In the Sham group, the cardiomyocytes were well arranged, the myocardial fiber structure was normal, and no degeneration or myocardial necrosis was observed (Fig. 4). However, in contrast to the Sham group, the
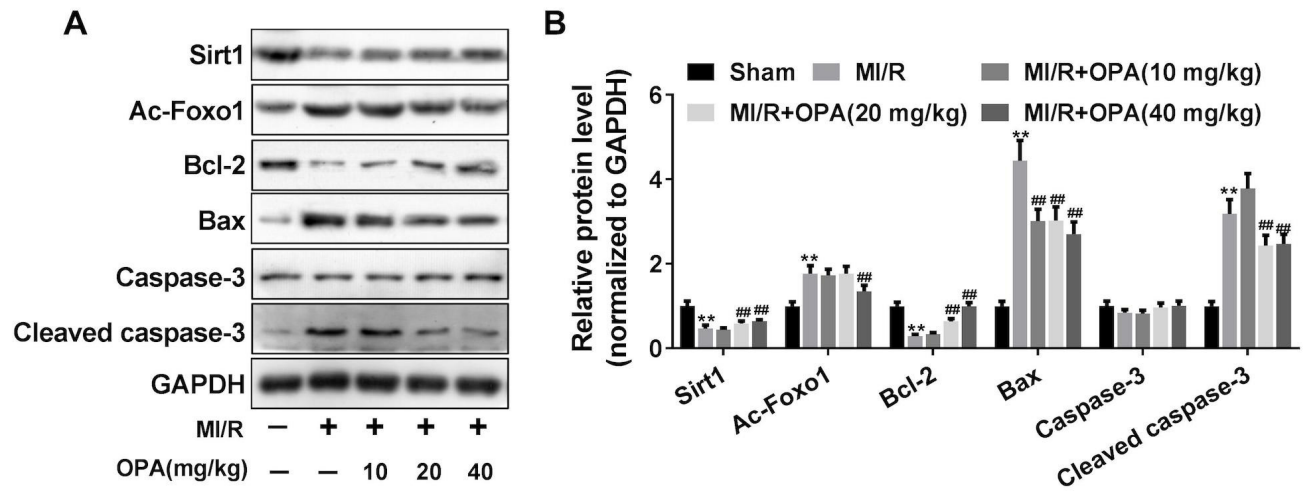

Figure 5. OPA activates the Sirt1/Foxo1 signaling pathway and inhibits the apoptosis of cardiomyocytes in MI/R mice.

(A) Representative images of the western blot results. (B) Quantitative densitometric analysis of the proteins. ${ }^{*} P<0.05,{ }^{* *} P<0.01 \mathrm{com}$ pared with the Sham group; ${ }^{P}<<0.05,{ }^{\#} P<0.01$ compared with the MI/R group. 

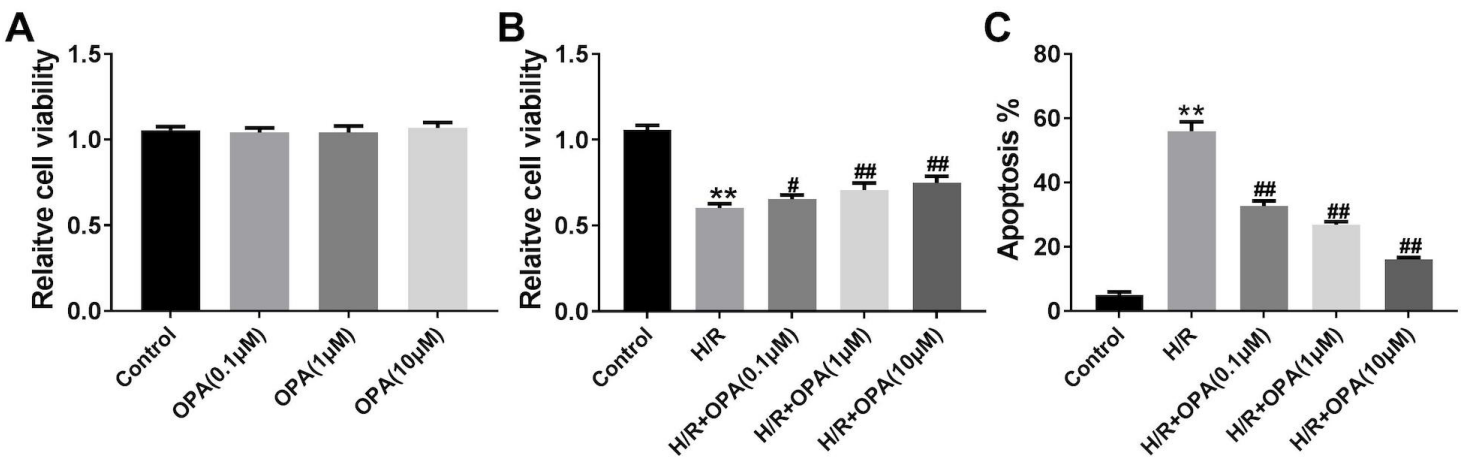

Figure 6. Effects of OPA on the viability and apoptosis of $\mathrm{H} / \mathrm{R}$-treated $\mathrm{H} 9 \mathrm{c} 2$ cells.

(A) The cell viability of $\mathrm{H} 9 \mathrm{C} 2$ cells without H/R treatment was measured by CCK-8 assay. (B) The cell viability of H/R-treated H9c2 cells was measured by CCK-8 assay. (C) Cell apoptosis was detected by flow cytometry assay. ${ }^{*} \mathrm{P}<0.05,{ }^{* *} P<0.01$ compared with the Control group; $\#<<0.05, \# P<0.01$ compared with the H/R group.

$\mathrm{MI} / \mathrm{R}$ group presented with ruptured myocardial fibers, karyopyknosis and fragmentation, and a large area of cardiac necrosis (Fig. 4). However, treatment with OPA significantly mitigated the MI/R-induced pathological alterations in a dose-dependent manner (Fig. 4). These data showed that OPA could protect cardiomyocytes from MI/R-induced necrosis.

\section{OPA activates the Sirt1/Foxo1 signaling pathway and inhibits the apoptosis of cardiomyocytes in MI/R mice}

Considering that the above results have clearly demonstrated that OPA can protect against the MI/R injury, we explored a potential mechanism that regulates this effect. We focused on the Sirt1/Foxo1-mediated apoptosis signaling pathway, which is related to the MI/R injury. Western blot assays (Fig. 5A-B) showed that Sirt1 expression was significantly decreased, but ac-Foxo1 expression was obviously increased in the $\mathrm{MI} / \mathrm{R}$ group compared with the Sham group. Notably, these effects induced by $\mathrm{MI} / \mathrm{R}$ were obviously reversed by OPA treatment; that is, Sirt1 expression significantly increased after OPA treatment, and ac-Foxo1 expression significantly decreased after OPA treatment. The detection of apoptosis-related proteins showed that compared with the mice subjected to $\mathrm{MI} / \mathrm{R}$ alone, the mice subjected to $\mathrm{MI} / \mathrm{R}$ and treated with OPA exhibited obviously decreased expression of Bax and cleaved caspase- 3 and increased expression of $\mathrm{Bcl}-2$, indicating that OPA could reduce the $\mathrm{MI} / \mathrm{R}$-induced apoptosis of cardiomyocytes.
Therefore, OPA could activate the Sirt1/Foxo1 signaling pathway and inhibit the apoptosis of cardiomyocytes in $\mathrm{MI} / \mathrm{R}$ mice.

\section{Effects of OPA on the viability and apoptosis of H/R- treated $\mathrm{H} 9 \mathrm{c} 2$ cells}

An $\mathrm{H} / \mathrm{R}$ injury model was established in $\mathrm{H} 9 \mathrm{c} 2$ cells to confirm the effects of OPA on MI/R injury and the underlying mechanism in vitro. A CCK-8 assay was performed to evaluate cardiomyocyte viability in the different groups. As shown in Fig. 6A, OPA treatment had no significant effect on the viability of cardiomyocytes in which $\mathrm{H} / \mathrm{R}$ was not induced. In Fig. $6 \mathrm{~B}$, compared to that of the control cells, the cell viability of the $H / R$ induced $\mathrm{H} 9 \mathrm{c} 2$ cells was decreased by approximately $45.61 \%$. Treatment with OPA markedly increased the $\mathrm{H} / \mathrm{R}$-mediated decrease in cell viability in a dose-dependent manner. Specifically, compared with that in the $\mathrm{H} / \mathrm{R}$ group, cell viability in the $\mathrm{H} / \mathrm{R}+\mathrm{OPA}(0.1 \mu \mathrm{M})$ group, $\mathrm{H} / \mathrm{R}+\mathrm{OPA}(1 \mu \mathrm{M})$ group and $\mathrm{H} / \mathrm{R}+\mathrm{OPA}(10 \mu \mathrm{M})$ group were increased by approximately $5.05 \%, 10.41 \%$ and $14.53 \%$, respectively. In addition, the flow cytometry results suggested that cell apoptosis in the $H / R$ group was significantly higher than that in the Control group by $50.84 \%$. Nevertheless, compared with that in the $\mathrm{H} / \mathrm{R}$ group, the cell viability in the $\mathrm{H} / \mathrm{R}+\mathrm{OPA}(0.1$ $\mu \mathrm{M})$ group, $\mathrm{H} / \mathrm{R}+\mathrm{OPA}(1 \mu \mathrm{M})$ group and $\mathrm{H} / \mathrm{R}+\mathrm{OPA}$ $(10 \mu \mathrm{M})$ group were decreased by approximately $23.26 \%$, $29.05 \%$ and $40.50 \%$, respectively (Fig. 6C). These results
A

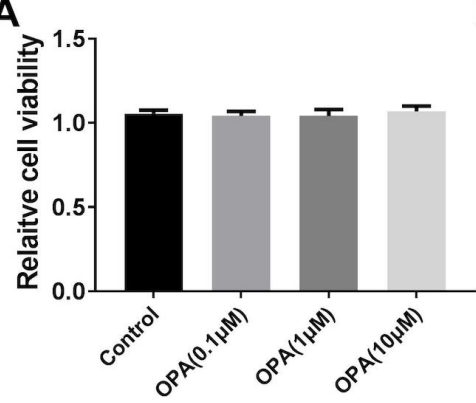

B

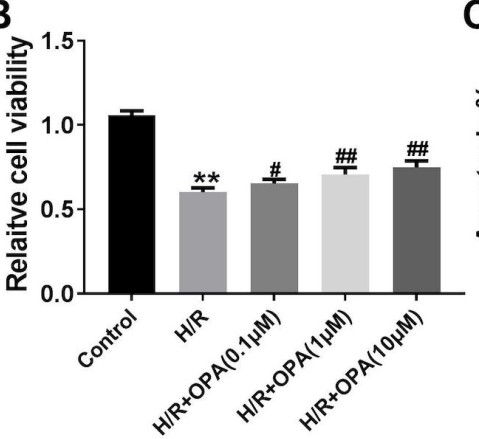

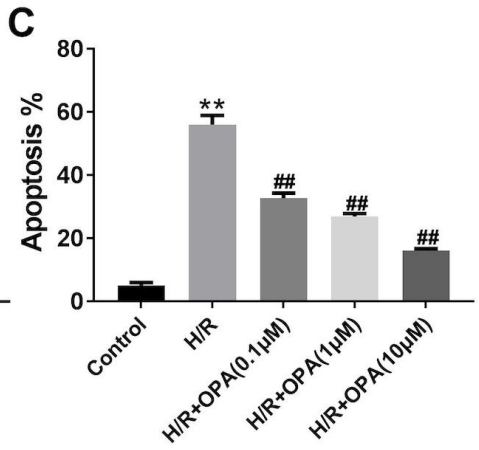

Figure 7. OPA activates the Sirt1/Foxo1 signaling pathway and inhibits the apoptosis of cardiomyocytes in H/R-induced H9c2 cells. (A) Representative images of the western blot results. (B) Quantitative densitometric analysis of the proteins. ${ }^{*} P<0.05$, ${ }^{* *} P<0.01$ compared with the Control group; ${ }^{\prime} P<0.05$, $\# P<0.01$ compared with the H/R group. 
indicated that OPA could increase the cardiomyocyte viability and inhibit the cardiomyocyte apoptosis induced by $\mathrm{H} / \mathrm{R}$ in vitro.

\section{OPA activates the Sirt1/Foxo1 signaling pathway and inhibits apoptosis of cardiomyocytes in H/R-treated H9c2 cells}

The effects of OPA on the expression levels of Sirt1, ac-Foxo1, Bcl2, Bax and cleaved Caspase 3 in H/R-treated $\mathrm{H} 9 \mathrm{C} 2$ cells were analyzed by western blot to elucidate the regulatory mechanism (Fig. 7A-B). Consistent with the in vivo results, the Sirt1 and $\mathrm{Bcl}-2$ levels were notably reduced, but the ac-FOXO1, Bax and cleaved Caspase-3 levels were significantly increased in the H/R group compared with the Control group. However, when compared with those in the Control group, the Sirt1 and Bcl-2 levels were notably increased but the ac-FOXO1, Bax and cleaved Caspase-3 levels were significantly decreased in the $\mathrm{H} / \mathrm{R}+\mathrm{OPA}(0.1 \mu \mathrm{M})$ group, $\mathrm{H} / \mathrm{R}+\mathrm{OPA}(1 \mu \mathrm{M})$ group and $\mathrm{H} / \mathrm{R}+\mathrm{OPA}(10 \mu \mathrm{M})$ group, indicating that OPA could promote the Sirt1/Foxo1 pathway and inhibit apoptosis of cardiomyocytes in an in vitro $\mathrm{MI} / \mathrm{R}$ injury model.

\section{DISCUSSION}

With the development of the social economy and changes in people's lifestyles, the risk factors for cardiovascular disease continue to increase. Tissue injury caused by myocardial ischemia is an important cause of fatal diseases and is commonly observed in clinical practice. Many mechanisms, including reactive oxygen species (ROS) production, inflammation, apoptosis, mitochondrial dysfunction, intracellular calcium overload, etc., are involved in the pathophysiological processes of myocardial ischemia/reperfusion injury (Song et al., 2017; Mastrocola et al., 2016; Jin et al., 2018). Understanding the complexity of pathological reperfusion injury and its mechanisms may lead to the development of a promising therapeutic strategy for cardiac I/R injury. In our current study, we found that OPA protected mouse hearts (in vivo) and $\mathrm{H} 9 \mathrm{c} 2$ cells (in vitro) against $\mathrm{I} / \mathrm{R}$ injury by regulating the Sirt1/Foxo1-mediated apoptosis pathway.

The mouse model of MI/ $\mathrm{R}$ was established by ligating the anterior descending branch of the left coronary artery, and the cardiac function, and hemodynamic and histopathological changes were evaluated. The EF and FS are important indicators of cardiac function (Bai et al., 2017). In this study, treatment with OPA significantly improved the EF and FS of the MI/R mice. Necrosis is also one of the most common forms of cell death in the cardiac tissue after I/R injury. Damage to the myocardial cell membrane leads to the release of myocardial enzymes and proteins, including CK-MB, cTnI and c'Tn'T, into the peripheral blood, and these enzymes and proteins are widely used as biomarkers of myocardial injury in clinical practice (Guo et al., 2019). Therefore, the levels of $\mathrm{CK}-\mathrm{MB}$, cTnI and cTnT in peripheral blood can reflect the degree of myocardial ischemia-induced necrosis. Here, OPA treatment reduced the MI/R-induced increase in the levels of CK-MB, cTnI and cTnT, indicating that OPA mitigated MI/R injury. Furthermore, the clear pathological changes in the myocardial tissue observed by H\&E staining also reflected the protective effect of OPA against MI/R injury.

Sirt1 is a member of the Sirtuin family. Sirtuins are class III histone deacetylated transferases that rely on nicotinamide adenine dinucleotide $(\mathrm{NAD}+)$ for deacetylation (Sundaresan et al., 2011). Sirt1 can regulate many transcription factors by deacetylation to extensively regulate various processes, such as cell survival, apoptosis, growth, metabolism and aging (Higashida et al., 2013). Mounting evidence has proven that Sirt1 is involved in MI/R injury (Ding et al., 2015; Potenza et al., 2019). In particular, Sirt1 regulates the downstream protein Foxo1 by deacetylation, which inhibits apoptotic pathways and protects against the MI/R-induced injury (Yang et al., 2013; Yamamoto et al., 2011; Yu et al., 2016). Consistent with previous research, the present study found that Sirt1 expression was significantly downregulated in the $\mathrm{MI} / \mathrm{R}$ group, which was accompanied by enhanced AcFoxo1 expression and increased cardiac apoptosis. Both, the in vivo and in vitro studies showed that OPA treatment promoted an obvious increase in Sirt1 expression and Foxo1 deacetylation. The activated Sirt1 pathway was also related to the increased expression of anti-apoptotic proteins (Bcl-2) and decreased expression of proapoptotic proteins (Bax and cleaved Caspase-3). Together, these data demonstrate that the myocardial protection achieved by OPA treatment is mediated by activating Sirt1 signaling, and OPA-induced Sirt1 activation can inhibit Foxo1 acetylation, thus promoting anti-apoptotic effects.

Based on the abundant evidence showing the protective effect of paeoniflorin (a structural analog of OPA) against MI/R injury (Chen et al., 2018; Han et al., 2016a; Wu et al., 2019), and the reported inhibitory effects of OPA on oxidative damage and inflammation in mesangial cells and LPS-stimulated RAW264.7 cells (Yoo et al., 2018a), we speculate that OPA may protect against the $\mathrm{MI} / \mathrm{R}$ injury. Our experiments successfully verified the above hypothesis and elucidated the mechanism of action by which OPA protects against MI/R injury, namely, by regulating the Sirt1/Foxo1-mediated apoptosis signals. However, whether this mechanism affects apoptosis that is caused by oxidative damage, mitochondrial dysfunction or inflammation remains to be further studied.

\section{CONCLUSION}

Overall, our findings revealed for the first time that OPA plays a protective role in MI/R injury. More importantly, the Sirt1/Foxo1-mediated apoptotic pathway was found to play a critical role in regulating the cardioprotective effects of OPA. These results emphasize that OPA may serve as a novel agent for the treatment of $\mathrm{MI} / \mathrm{R}$ injury in clinical cardiac surgery and ischemic heart diseases.

\section{Acknowledgments}

Not applicable.

\section{Funding}

Not applicable.

\section{Availability of data and materials}

The datasets used and/or analyzed during the current study are available.

\section{Ethics approval and consent to participate}

All the procedures were performed in accordance with the Guidelines for the Care and Use of Laboratory Animals published by the U.S. National Institutes of Health (NIH publication NO. 85-23, revised in 1996). The protocol was reviewed and approved by the Ethical Com- 
mittee of ZheJiang Chinese Medical University before the animal study (No. ZSLL-2017-059).

\section{REFERENCES}

Bai R, Yin X, Feng X, Cao Y, Wu Y, Zhu Z, Li C, Tu P, Chai X (2017) Corydalis hendersoni Hemsl. protects against myocardial injury by attenuating inflammation and fibrosis via NF-xB and JAK2STAT3 signaling pathways. J Ethnopharmacol 207: 174-183. https:// doi.org/10.1016/j.jep.2017.06.020

Buja L. M. J. T. H. I. J. (2013) The pathobiology of acute coronary syndromes: clinical implications and central role of the mitochondria. Tex Heart Inst J 40: 221-228.

Chen C, Du P, Wang J (2015) Paeoniflorin ameliorates acute myocardial infarction of rats by inhibiting inflammation and inducible nitric oxide synthase signaling pathways. Mol Med Rep 12: 3937-3943. https://doi.org/10.3892/mmr.2015.3870

Chen H, Dong Y, He X, Li J, Wang J (2018) Paeoniflorin improves cardiac function and decreases adverse postinfarction left ventricular remodeling in a rat model of acute myocardial infarction. Drug Design Develop Therapy 12: 823-836. https://doi.org/10.2147/DDDT. $\mathrm{S} 163405$

Chen Y-F, Wu K-J, W Wood G (2013) Paeonia lactiflora extract attenuating cerebral ischemia and arterial intimal hyperplasia is mediated by paeoniflorin via modulation of VSMC migration and Ras/MEK/ ERK signaling pathway. Evid Based Complement Alternat Med 2013: 482428. https:// doi.org/10.1155/2013/482428

Ding M, Lei J, Han H, Li W, Qu Y, Fu E, Fu F, Wang X (2015) SIRT1 protects against myocardial ischemia-reperfusion injury via activating eNOS in diabetic rats. Cardiovascular Diabetol 14: 143-147. https://doi.org/10.1186/s12933-015-0299-8

Feng C, Liu M, Shi X, Yang W, Kong D, Duan K, Wang Q (2010) Pharmacokinetic properties of paeoniflorin albiflorin and oxypaeoniflorin after oral gavage of extracts of Radix Paeoniae Rubra and Radix Paeoniae Alba in rats. J Ethnopharmacol 130: 407-413. https:// doi.org/10.1016/j.jep.2010.05.028

Finegold JA, Asaria P, Francis DP (2013) Mortality from ischaemic heart disease by country region and age: Statistics from World Health Organisation and United Nations. Int J Cardiol 168: 934-945. https://doi.org/10.1016/j.ijcard.2012.10.046

Guan X-H, Liu X-H, Hong X, Zhao N, Xiao Y-F, Wang L-F, Tang L, Jiang K, YQian Y-S, Deng K-Y (2016) CD38 deficiency protects the heart from ischemia/reperfusion injury through activating SIRT1/FOXOs-mediated antioxidative stress pathway. Oxid Med Cell Longev 2016: 7410257. https://doi.org/10.1155/2016/7410257

Guo C, Zhang J, Zhang P, Si A, Zhang Z, Zhao L, Lv F, Zhao G (2019) Ginkgolide B ameliorates myocardial ischemia reperfusion injury in rats via inhibiting endoplasmic reticulum stress. Drug Design Develop Therapy 13: 767. https://doi.org/10.2147/DDDT.S179101

Han F, Zhou D, Yin X, Sun Z, Han J, Ye L, Zhao W, Zhang Y, Wang Z, Zheng L (2016a) Paeoniflorin protects diabetic mice against myocardial ischemic injury via the transient receptor potential vanilloid 1/calcitonin gene-related peptide pathway. Cell Bioscience 6: 37-42. https://doi.org/10.1186/s13578-016-0085-7

Higashida K, Kim SH, Jung SR, Asaka M, Holloszy JO, Han D-H (2013) Effects of resveratrol and SIRT1 on PGC- $1 \alpha$ activity and mitochondrial biogenesis: a reevaluation. PLoS Biol 11: e1001603. https://doi.org/10.1371/journal.pbio.1001603

Jin Q, Li R, Hu N, Xin T, Zhu P, Hu S, Ma S, Zhu H, Ren J, Zhou H (2018) DUSP1 alleviates cardiac ischemia/reperfusion injury by suppressing the Mff-required mitochondrial fission and Bnip3related mitophagy via the JNK pathways. Redox Biol 14: 576-587. https://doi.org/10.1016/j.redox.2017.11.004

Li J-Z, Yu S-Y, Wu J-H, Shao QR, Dong X-M (2012) Paeoniflorin protects myocardial cell from doxorubicin-induced apoptosis through inhibition of NADPH oxidase. Can J Physiol Pharmacol 90: 1569-1575. https://doi.org/10.1139/y2012-140

Li X, Yang R, Yang X, Zheng E (2017) Paeonol protects H9C2 cardiomyocytes from ischemia/reperfusion injury by activating Notch1 signaling pathway in vitro. Medine 10: 2866-2873. https://doi. org/10.1038/srep23693

Mastrocola R, Penna C, Tullio F, Femminò S, Nigro D, Chiazza F, Serpe L, Collotta D, Alloatti G, Cocco M (2016) Pharmacological inhibition of NLRP3 inflammasome attenuates myocardial ischemia/reperfusion injury by activation of RISK and mitochondrial pathways. Oxidativ Med Cell Longevity 2016: 5271251 https://doi. org/10.1155/2016/5271251

Nizamutdinova IT, Jin YC, Kim JS, Yean MH, Kang SS, Kim YS, Lee JH, Seo HG, Kim HJ, Chang KC (2008) Paeonol and paeoniflorin the main active principles of Paeonia albiflora protect the heart from myocardial ischemia/reperfusion injury in rats. Planta Med 74: 14 18. https://doi.org/10.1055/s-2007-993775

Potenza MA, Sgarra L, Nacci C, Leo V, De Salvia MA, Montagnani M (2019) Activation of AMPK/SIRT1 axis is required for adiponectin- mediated preconditioning on myocardial ischemia-reperfusion (I/R) injury in rats. PloS One 14: e0210654. https://doi.org/ 10.1371/journal.pone.0210654

Qian G-Q, Ding J, Zhang X, Yin X, Gao Y, Zhao G-P (2015) Preconditioning with glycyrrhizic ferulic paeoniflorin cinnamic prevents rat hearts from ischemia/reperfusion injury via endothelial nitric oxide pathway. Pharmacogn Mag 11: 292-296. https://doi.org/ 10.4103/0973-1296.153081

Salas MA, Valverde CA, Sánchez G, Said M, Rodriguez JS, Portiansky EL, Kaetzel MA, Dedman JR, Donoso P, Kranias EG (2010) The signalling pathway of CaMKII-mediated apoptosis and necrosis in the ischemia/reperfusion injury. I Mol Cell Cardiol 48: 1298-1306. https://doi.org/10.1016/j.yjmcc.2009.12.015

Song $\mathrm{H}$, Yan C, Tian X, Zhu N, Li Y, Liu D, Liu Y, Liu M, Peng C, Zhang Q (2017) CREG protects from myocardial ischemia/reperfusion injury by regulating myocardial autophagy and apoptosis. Biochim Biophys Acta (BBA)-Molecular Basis of Disease 1863: 1893-1903. https://doi.org/10.1016/j.bbadis.2016.11.015

Sundaresan NR, Pillai VB, Gupta MP (2011) Emerging roles of SIRT1 deacetylase in regulating cardiomyocyte survival and hypertrophy. J Mol Cell Cardiol 51: 614-618. https://doi.org/10.1016/j. yimcc.2011.01.008

Wang J-X, Zhang XJ, Li Q, Wang K, Wang Y, Jiao JQ, Feng C, Teng S, Zhou LY, Gong YJ (2015) MicroRNA-103/107 regulate programmed necrosis and myocardial ischemia/reperfusion injury through targeting FADD. Circ Res 117: 352-363. https://doi. org/10.1161/CIRCRESAHA.117.305781

Wang K, Liu F, Liu C, An T, Zhang J, Zhou L, Wang M, Dong Y, $\mathrm{Li}$ N, Gao JJ (2016) The long noncoding RNA NRF regulates programmed necrosis and myocardial injury during ischemia and reperfusion by targeting miR-873. Cell Death Differ 23: 1394. https://doi. org/10.1038/cdd.2016.28

Wang X, Yuan B, Cheng B, Liu Y, Zhang B, Wang X, Lin X, Yang B, Gong GJ (2019) Crocin alleviates myocardial ischemia/reperfusioninduced endoplasmic reticulum stress via regulation of miR-34a/ Sirt1/Nrf2 pathway. Shock 51: 123-130. https://doi.org/10.1097/ SHK.0000000000001116

Wu F, Ye B, Wu X, Lin X, Li Y, Wu Y, Tong L (2019) Paeoniflorin on rat myocardial ischemia reperfusion injury of protection and mechanism research. Pharmacology 105: 281-288 https://doi. org/10.1159/000503583

Xu T, Ding W, Ao X, Chu X, Wan Q, Wang Y, Xiao D, Yu W, Li M, Yu FJ (2019) ARC regulates programmed necrosis and myocardial ischemia/reperfusion injury through the inhibition of $\mathrm{mPTP}$ opening. Redox Biol 20: 414-426. https://doi.org/10.1016/j.redox.2018.10.023

Yamamoto T, Sadoshima J (2011) Protection of the heart against ischemia/reperfusion by silent information regulator 1 . Trends Cardiovascul Med 21: 27-32. https://doi.org/10.1016/j.tcm.2012.01.005

Yang Y, W. Duan Y. Lin W. Yi Z. Liang J. Yan N. Wang C. Deng S. Zhang Y. Li (2013) SIRT1 activation by curcumin pretreatment attenuates mitochondrial oxidative damage induced by myocardial ischemia reperfusion injury. Free Radic Biol Med 65: 667-679. https:// doi.org/10.1016/j.freeradbiomed.2013.07.007

Yoo C-K, Hwang JH, Lee K, Lee Y-J, Kim KJ, Lee BY (2018a) Antiinflammatory effects of moutan cortex radicis extract paeoniflorin and oxypaeoniflorin through TLR signaling pathway in RAW264. 7 cells. J Food Nutrit Res 6: 26-31. https://doi.org/10.12691/jfnr-6-1-5 Yu L, Q. Li B. Yu Y. Yang Z. Jin W. Duan G. Zhao M. Zhai L. Liu D. Yi (2016) Berberine attenuates myocardial ischemia/reperfusion injury by reducing oxidative stress and inflammation response: role of silent information regulator 1. Oxi Medi Cell Longevity 2016: https://doi.org/10.1155/2016/1689602

Yu L, Sun Y, Cheng L, Jin Z, Yang Y, Zhai M, Pei H, Wang X, Zhang $\mathrm{H}$, Meng QJ (2014) Melatonin receptor-mediated protection against myocardial ischemia/reperfusion injury: role of SIRT 1. Redox Biol 57: 228-238. https://doi.org/10.1111/jpi.12161

Zhang B, Zhai M, Li B, Liu Z, Li K, Jiang L, Zhang M, Yi W, Yang J, Yi D (2018) Honokiol ameliorates myocardial ischemia/reperfusion injury in type 1 diabetic rats by reducing oxidative stress and apoptosis through activating the SIRT1-Nrf2 signaling pathway. Oxi Med Cell Longevity 2018: https://doi.org/10.1155/2018/3159801

Zhang M-H, Feng L, M. Zhu MM, Gu JF, Wu C, Jia XB (2013) Antioxidative and anti-inflammatory activities of paeoniflorin and oxypaeoniflora on AGEs-induced mesangial cell damage. Planta Med 79: 1319-1323. https://doi.org/10.1055/s-0033-1350649

Zhu K-F, Wang YM, Zhu JZ, Zhou QY, Wang NF (2016) National prevalence of coronary heart disease and its relationship with human development index: a systematic review. Eur J Prev Cardiol 23: 530-543. https://doi.org/10.1177/2047487315587402

Zhu L, Wei T, Gao J, Chang X, He H, Luo F, Zhou R, Ma C, Liu $\mathrm{Y}$, Yan TJA (2015) The cardioprotective effect of salidroside against myocardial ischemia reperfusion injury in rats by inhibiting apoptosis and inflammation. Apoptosis 20: 1433-1443. https://doi. org/10.1007/s10495-015-1174-5 